\title{
Endoscopic anatomical study on anterior communicating artery aneurysm surgery by endonasal transphenoidal approach
}

\author{
Junwei Ma ${ }^{1}$, Zhimin Wang ${ }^{1 *}$, Niankai Zhang ${ }^{2}$, Shengshan $\mathrm{Li}^{3}$, Dongyi Jiang ${ }^{1}$ and Hanchun Chen ${ }^{1}$
}

\begin{abstract}
Background: Endonasal transphenoidal approach by neuroendoscopy has its own advantage, such as direct access, invasive, better visualization of the anterior communicating artery aneurysm and so on. The study is to provide anatomical knowledge for anterior communicating artery aneurysm surgery by endonasal transphenoidal approach with neuroendoscopy.
\end{abstract}

Materials: Take 10 skull base specimens, observe and measure the anatomical structures around anterior communicating artery. Take 10 cadaveric heads, simulate the anterior communicating artery aneurysm surgery with neuroendoscopy by endonasal transphenoidal approach. Find the natural opening of sphenoid sinus, then open the skull base, expand bone window in anterior skull base. After that, cut off the dura, find the optic nerve, optic chiasm, cisterna lamina terminalis, anterior cerebral artery, a portion of frontal lobe, anterior communicating artery complex and its important branches, such as heubner artery, hypothalamic artery, orbitofrontal artery and so on. Lift up anterior communicating artery complex and seperate arachnoid in cisterna lamina terminalis, the lamina terminalis is exposed. Block bilateral A1 of anterior cerebral artery with aneurysm clip, the anterior communicating artery complex and its important branches are in view, so we can clip anterior communicating artery aneurysm safely.

Results: Anterior communicating artery aneurysm surgery can be finished with neuroendoscopy by endonasal transphenoidal approach. The vital structures can be clearly observed with neuroendoscopy. The rhombus anatomic region formed by bilateral olfactory nerve and optic nerve is a safe surgical area. According to the calculation, the safe surgical area is about $161.48 \pm 12.78 \mathrm{~mm}^{2}$. Measure the distance between the important anatomic structures in the rhombus anatomic region. By means of SPSS 17 Statistical analysis software, the measuring distance is expressed with $(x \pm S) m m$.

Conclusion: The anterior communicating aneurysm surgery by endonasal transphenoidal approach with neuroendoscopy has its own advantage, such as direct access, minimally invasive, less bleeding, light pain, quick recovery, better visualization of the anterior communicating artery aneurysm and so on. This operation approach needs further study and exploration to clinical application, in order to become a mature approach of anterior communicating artery aneurysm surgery.

Keywords: Endoscope, Anterior communicating artery, Cerebral aneurysm, Anatomy

\footnotetext{
*Correspondence: wzhimin@hotmail.com

'Department of Neurosurgery, Suzhou Kowloon Hospital Affiliated Shanghai

Jiao Tong University, Suzhou 266021, China

Full list of author information is available at the end of the article
} 


\section{Background}

Endovascular techniques and craniotomy play an important role in the treatment of anterior communicating artery aneurysm. Although they are very mature, both of them have disadvantages, such as surgical injury, incomplete clipping, infection, hydrocephaly or even serious complications sometimes. However, endonasal transphenoidal approach with neuroendoscopy has its own advantage, such as direct access, minimally invasive, less bleeding, light pain, quick recovery, better visualization of the anterior communicating artery aneurysm and so on [1-3]. Besides, endonasal transphenoidal approach with neuroendoscopy is more minimally invasive and can reduce postoperative complications. The study is to provide anatomical basis for endoscopic endonasal transphenoidal approach for anterior communicating artery aneurysm surgery, and then apply to clinical. Anterior communicating artery complex has many important physiologicalfunctions, so the anterior communicating artery aneurysm surgery is a very difficult aneurysm surgery. For its postoperative complications, the anterior communicating artery aneurysm has been a focus in clinics. As the neuroendoscopic surgery become mature, we know more and more about the complex anatomy of skull base. By anatomical study on the cadaveric heads, we find that the anterior communicating artery complex and its surrounding vital structures can be clearly observed with neuroendoscopy. So we have a series anatomical study on skull base, which maybe helpful for anterior communicating artery aneurysm surgery.

\section{Methods}

Take 10 cadaveric heads perfused with latex and 10 skull base specimens

DELON Neuroendoscopy produced by Shanghai Delon Special Rivet Manufacture Co.,Ltd. McGrady brand aneurysm clip and clamp produced by Aesculap AG \& Co. KG in Germany. Calipers and Protractor produced by Shanghai DELI Company.

\section{Observation and measurement on skull base specimens} Take 10 skull base specimens, for CT scans (Fig. 1), then observe the anatomical structures around anterior communicating artery complex. Measure the distance between the vital anatomical landmarks with caliper, and then measure the angle formed by bilateral optic nerve and the olfactory nerve with protractor. By means of SPSS 17 Statistical analysis software, the results are expressed with $(x \pm S) \mathrm{mm}$. The distance between the bilateral olfactory nerve in the juncture of cerebral falx and dura mater. The distance between the bilateral optic nerve in the internal foramen of the optic canal. The distance from frontier edge of optic chiasma to upper edge of optic nerve impression. The distance between the two junctions of olfactory nerve and optic nerve. The distance between the two junctions connection and the juncture of cerebral falx and cranial dura mater. The distance between the two junctions connection and optic nerve pressure trace. The length of A1.

\section{Simulate anterior communicating artery aneurysm surgery with neuroendoscopy by endonasal transphenoidal approach}

Simulate the neuroendoscopic surgery, keep the cadaveric heads slightly backwards, which fixed on the shelves. Start the surgery from bilateral nasal with neuroendoscopy. Firstly, find the middle turbinate, we can see the upper turbinate above the middle turbinate. The recessus sphenoethmoidalis and sphenoid natural openings is at the back of the upper turbinate (Fig. 3). Resect the anterior wall of sphenoid sinus, we can see the optic nerve carina, carotid artery carina, carotid artery optic nerve crypt and slopes (Fig. 4). Secondly, open and expand the base of skull bone in the middle of two carotid artery optic nerve crypts, forming the bone window with a diameter of about $12 \mathrm{~mm}$ (Fig. 5). Then we can see the dural and intercavernous sinus, cut off the dura to the edge of the bone window, the arachnoid in the chiasmatic cistern and interhemispheric cistern appears. Separate the arachnoid, we clearly see the anterior communicating artery complex and its vital anatomic structures (Figs. 6 and 7). Thirdly, simulate the anterior communicating artery aneurysm surgery, at the same time, expose the structures around the anterior communicating artery aneurysm, and then observe and record them (Figs. 8 and 9).

\section{Results}

Anatomic observation and measurement

The rhombus anatomic region is formed by bilateral olfactory nerve and optic nerve, which is a very safe

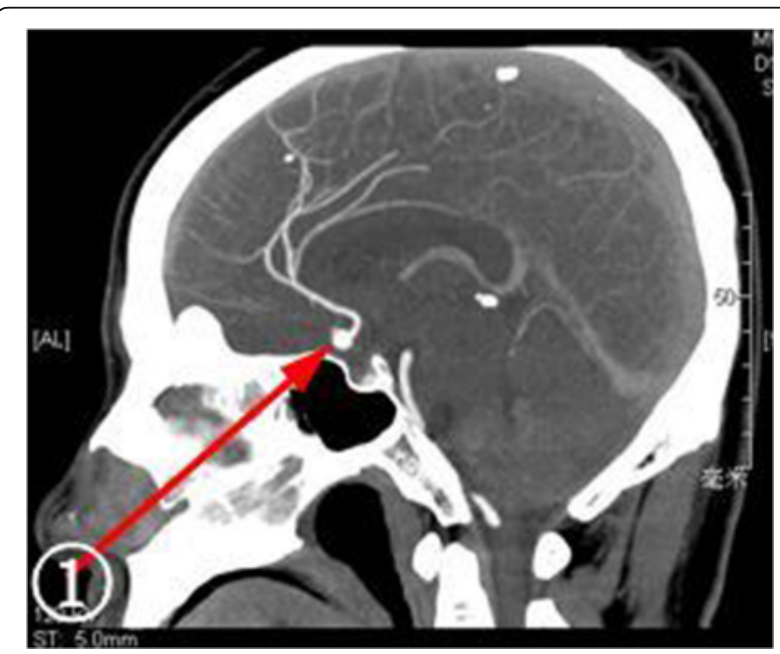

Fig. 1 Red arrow shows anatomical approach 
surgical area. Bilateral olfactory nerve is at lateral of bilateral optic nerve, and the fila olfactoria branched off from olfactory bulb which passes through ethmoidal forame in front of the juncture of cerebral falx and cranial dura mater distributes to the nasal mucosa (Fig. 2).

Diagram 1: The angle formed by bilateral olfactory nerve and optic nerve in the rhombus anatomic region. $(\bar{x} \pm \mathrm{s})$

\begin{tabular}{ll}
\hline Angle formed by nerve (Fig. 2) & Result $\left(^{\circ}\right)$ \\
The angel formed by bilateral optic nerve & $77.76 \pm 7.98$ \\
The angle formed by bilateral olfactory nerve & $45.78 \pm 3.68$ \\
\hline
\end{tabular}

Diagram 2: The distance between the important anatomic structures in the rhombus anatomic region. $(\bar{x} \pm s)$

The distance between the ralated anatomic structures

Result (mm)

(Fig. 2)

The distance between the bilateral olfactory nerve in the $8.23 \pm 1.34$ juncture of cerebral falx and dura mater.(a)

The distance between the bilateral optic nerve in the $\quad 14.32 \pm 3.13$ internal foramen of the optic canal.(d)

The distance from frontier edge of optic chiasma to upper edge of optic nerve impression.(e)

The distance between the two junctions of olfactory nerve and optic nerve.(c)

$6.12 \pm 2.23$

$15.36 \pm 2.12$

The distance between the two junctions connection and the juncture of cerebral falx and cranial dura mater.(b)

The distance between the two junctions connection and $\quad 5.42 \pm 0.23$ Optic nerve pressure trace.(f)

The length of $A 1$

$14.42 \pm 2.23$

According to the numerical calculation, the secure surgical area is about $161.48 \pm 12.78 \mathrm{~mm} 2$.

\section{Simulate the anterior communicating artery aneurysm surgery by endonasal transphenoidal approach with neuroendoscopy \\ Open the anterior wall of sphenoid sinus}

Find the middle turbinate with $0^{\circ}$ neuro-endoscopy, we can see the superior turbinate above the middle turbinate. The recessus sphenoethmoidalis and sphenoid natural opening is at the back of the superior turbinate, resect the anterior wall of sphenoid sinus. After that we can see the optic nerve bulge, carotid artery bulge, carotid artery optic nerve recess and the slope. In fact, we can see the carotid artery optic nerve recess or optic nerve canal bulge in 8 cadaveric heads. The midpoint of the bilateral carotid artery optic nerve recess is related to the optic nerve trace, which can help us open skull base accurately and safely. But in the other 2 cadaveric heads, we can not see the important anatomic

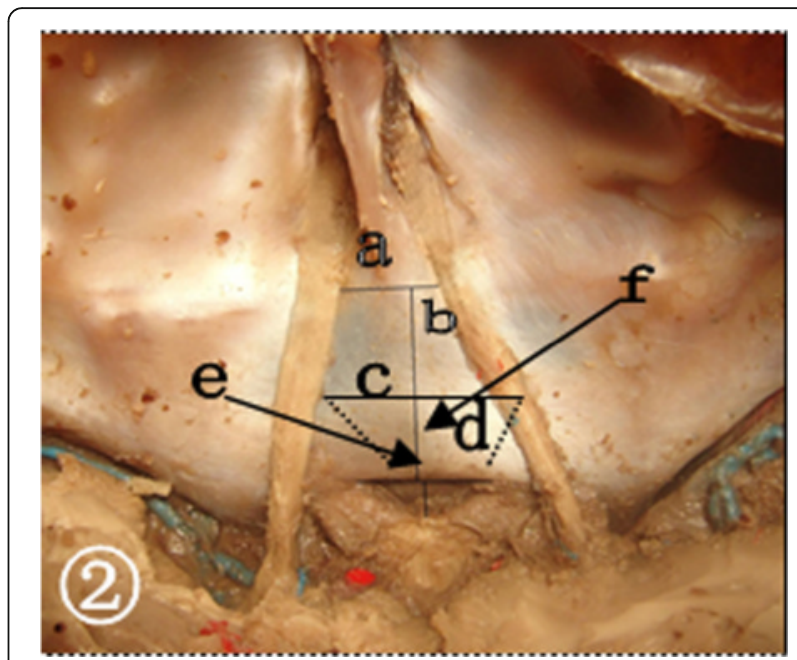

Fig. 2 Anterior skull base specimen with optic nerve and olfactory nerve. a The distance between the bilateral olfactory nerve in the juncture of cerebral falx and cranial dura mater. $\mathbf{d}$ The distance between the bilateral optic nerve in the anterior foramen of the optic canal. e The distance between leading edge of optic chiasma and upper edge of optic nerve impression. c The distance between the two junctions of olfactory nerve and optic nerve. $\mathbf{b}$ The distance between the two junctions connection and the juncture of cerebral falx and cranial dura mater. $\mathbf{f}$ The distance between the two junctions connection and optic pressure trace

marks. We can only see the slope and sellar with neuroendoscopy. With the help of the slope and sellar, we can determine the midline, the other line is the boundary of anterior skull base and sellar anterior wall. The intersection of the two lines is called the anchor point, which is related to the optic nerve trace, it can help us open skull base accurately and safely.

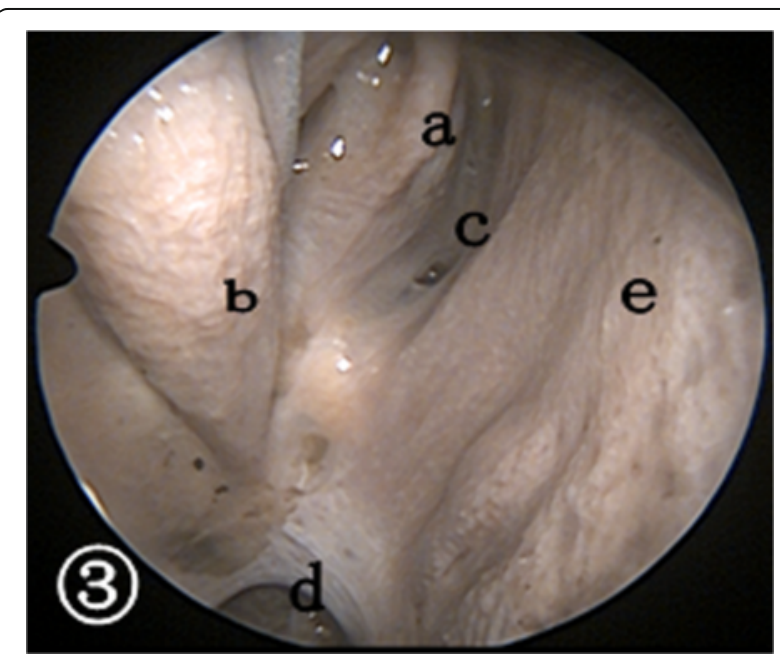

Fig. 3 Nasal structures. a Superior turbinate b Middle turbinate c Sphenoid sinus opening d Choanal e Nasal septum 


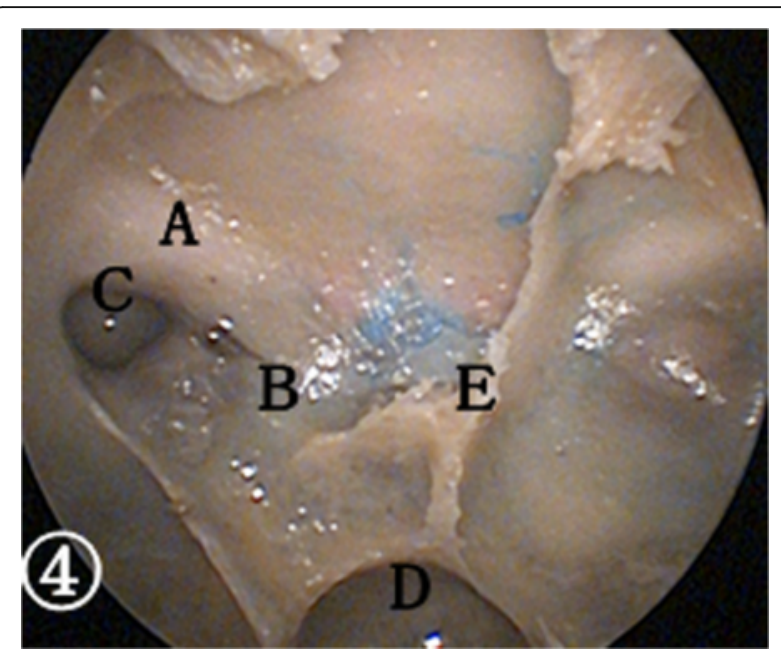

Fig. 4 Sphenoid sinus structures. a Optic nerve canal carina b Carotid artery carina c carotid artery optic nerve crypt. d Slope e Sphenoid sinus interval

\section{Open the anterior skull base}

With the help of the anchor point, we can open the anterior skull base forming the bone window for a diameter of $12 \mathrm{~mm}$. Then we can see the sellar dura easily, cut it and seperate arachnoid, several important anatomical structures are exposed. By $30^{\circ}$ neuroendoscopy, we can see optic nerve, optic chiasm, cisterna lamina terminalis, anterior cerebral artery, a portion of frontal lobe, anterior communicating artery complex and its important branches, such as heubner artery, hypothalamic artery, orbitofrontal artery and so on. Lift up anterior communicating artery complex and seperate arachnoid in cisterna lamina terminalis, the lamina terminalis is exposed. Then we simulate the anterior communicating aneurysm surgery, block bilateral A1 of

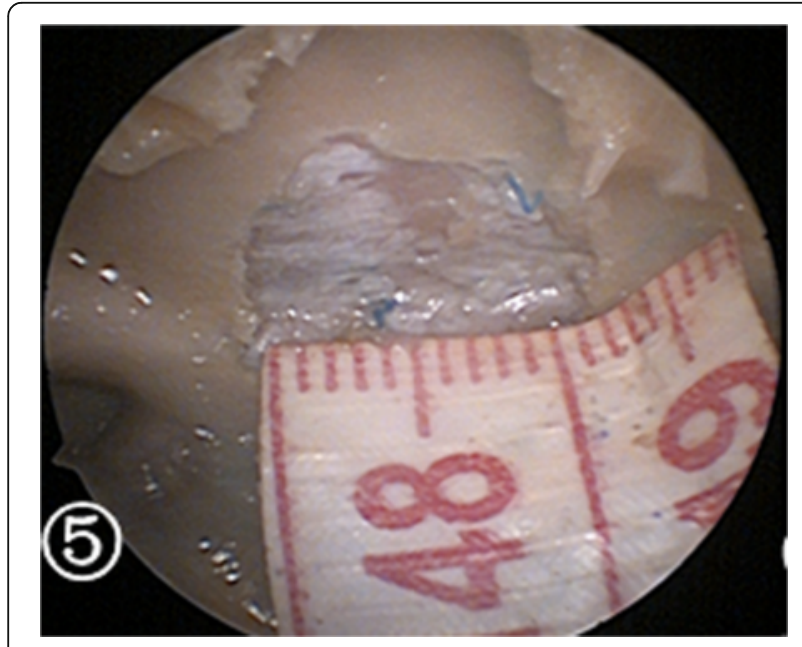

Fig. 5 The position and size of skull base window

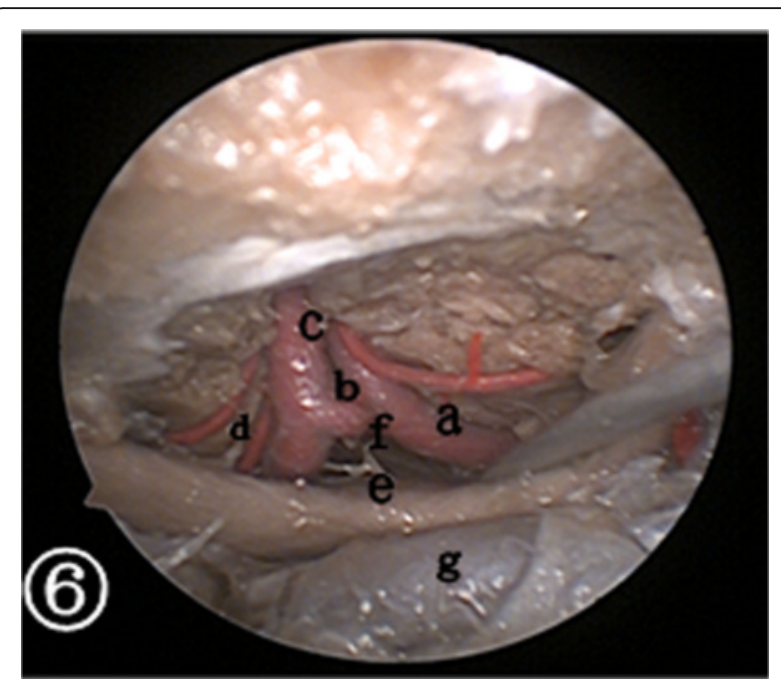

Fig. 6 The structures around anterior communicating artery complex. $\mathbf{a}$ A1 of anterior cerebral artery $\mathbf{b}$ anterior communicating artery $\mathbf{c}$ A2 of anterior cerebral artery $\mathbf{d}$ Heubner artery e optic chiasma $\mathbf{d}$ lamina terminalis $\mathbf{f}$ and $\mathbf{g}$ Pituitary

anterior cerebral artery with aneurysm clip, the anterior communicating artery complex and its important branches are in view, so we can clip anterior communicating artery aneurysm safely. After that we open lamina terminalis, this can lower the incidence of hydrocephaly [4].

\section{Expand bone window in anterior skull base}

Resect ethmoid sinus and the rear of the nasal septum along optic nerve canal to expand bone window in

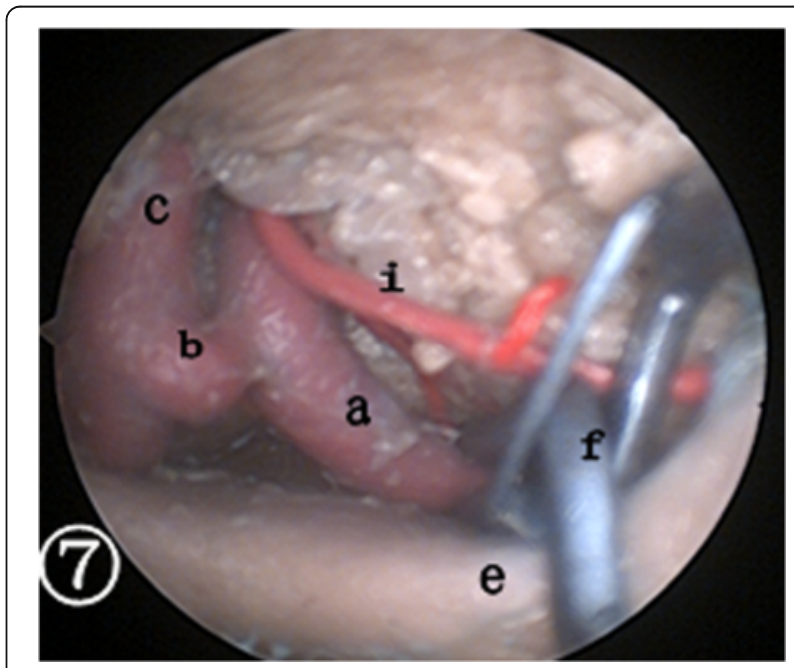

Fig. 7 The structures after clipping anterior communicating artery aneurysm. a A1 of anterior cerebral artery $\mathbf{b}$ anterior communicating artery $\mathbf{c}$ A2 of anterior cerebral artery $\mathbf{e}$ optic chiasma $\mathbf{f}$ aneurysm clip i orbitofrontal artery 


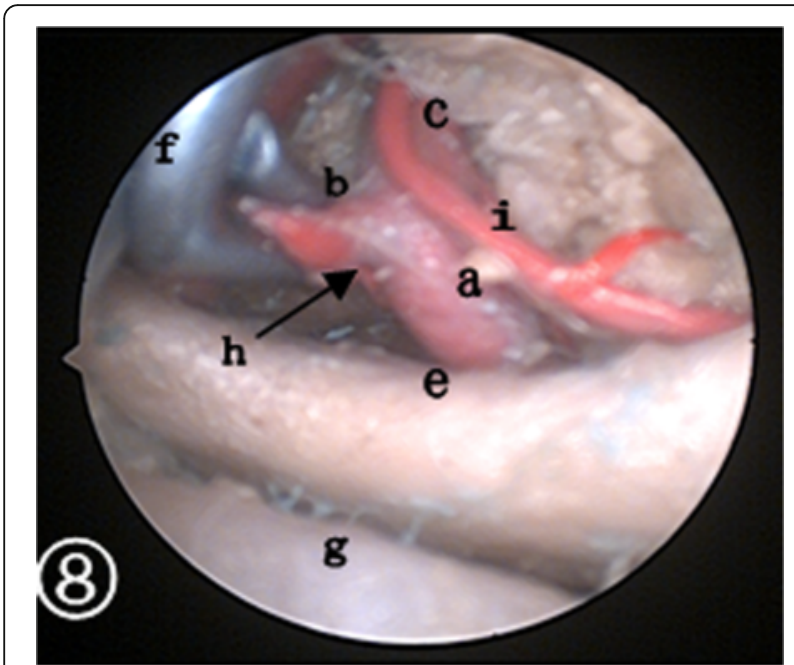

Fig. 8 The structures after clipping anterior communicating artery aneurysm. a A1 of anterior cerebral artery $\mathbf{b}$ anterior communicating artery c A2 of anterior cerebral artery e optic chiasma $\mathbf{f}$ aneurysm clip $\mathbf{h}$ hypothalamic artery $\mathbf{i}$ orbitofrontal artery $\mathbf{g}$ Pituitary

anterior skull base. Cut off dural $10 \mathrm{~mm}$ before optic chiasma, we can see olfactory nerve. Expand bone window along olfactory nerve until $22 \mathrm{~mm}$ before optic chiasma, and then cut off dural, the olfactory bulb is exposed. The bilateral olfactory nerve and optic nerve can form a rhombic region, in which we can operate safely. This is consistent with some reports [5]. If something unexpected happened in surgery, we can expand the bone window in anterior skull base along olfactory nerve to control intraoperative bleeding. After clipping

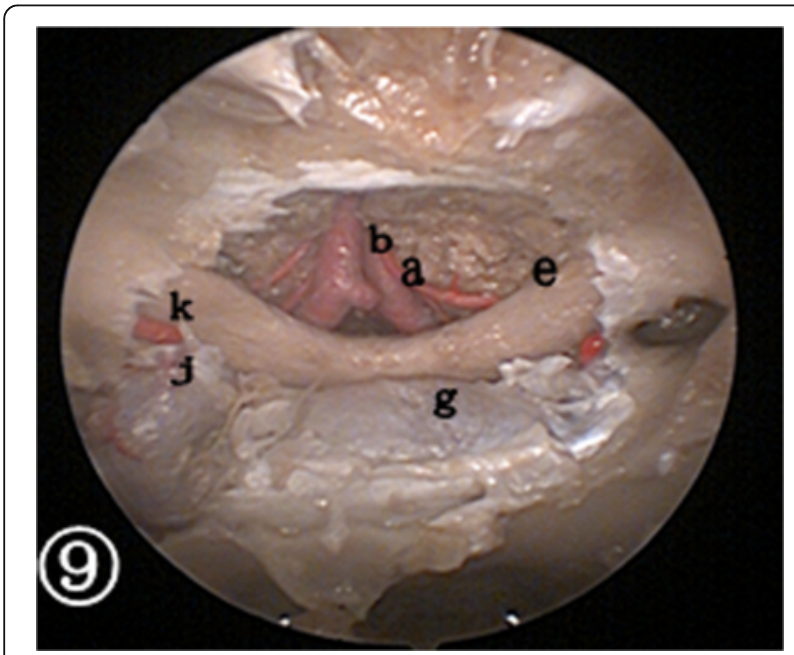

Fig. 9 The structures after extended resection of bone window. a A1 of anterior cerebral artery $\mathbf{b}$ anterior communicating artery $\mathbf{e}$ optic nerve $\mathbf{g}$ Pituitary j Carotid artery k ophthalmic artery the aneurysm, we can observe that the aneurysm is clipped completely with $30^{\circ}$ and $70^{\circ}$ neuroendoscopy.

\section{Discussion}

The study provide the anatomic basis for endonasal transphenoidal approach of anterior communicating artery aneurysm clipping by neuroendoscopy. The approach is significant and can be applied to clinic widely for several reasons: Firstly, endonasal transphenoidal approach by neuroendoscopy is more minimally invasive, and it can both relieve the pain and shorten the hospitalization time, most of all, it can reduce the postoperative complications. Secondly, neuroedoscopy can provide a more broad and clear surgical field, which can be safer than microscope operative surgery. Thirdly, the rupture of intracranial aneurysm occupies the third in cerebral vascular accident, second to cerebral thrombosis and cerebral hemorrhage of hypertension. According to statistics, more than $80 \%$ of spontaneous subarachnoid hemorrhage is caused by aneurysm rupture. While accident of anterior communicating artery aneurysm is about $35 \%$ of intracranial aneurysms [6,7].

Anterior communicating artery has important physiological functions, it can balance and compensate blood flow between bilateral hemispheres. Anterior communicating artery is adjacent to the third ventricle, hypothalamus, optic chiasm, and Heubner artery and other important structures. If any important structure injured in surgery, it can lead to serious complications, such as dysfunction, coma or even death. The most popular craniotomy approach for anterior communicating artery aneurysm is pterional and interhemispheric approach. Pterional approach is fit for exposing A1 and blocking blood flow conveniently in surgery. Interhemispheric approach is fit for anterior communicating artery aneurysm pointing to the rear and front. Both of them have their limitations and surgical injury. The pterional requires separation of sylvian fissure, pulling the frontal lobe, or even removal of part gyrus rectus to complete the surgery, and damage to gyrus rectus can cause the recent memory impairment [8]. Li JP, Zhao JZ and Wang S report 59 patients with surgical clipping of anterior communicating artery aneurysm of which $12(20.34 \%)$ patients have different parts of the brain tissue infarction [9].

In recent years, as the endoscopic techniques become mature and the equipments improve better, we find that transsphenoidal surgery is much better than craniotomy [10], and begin to resect sellar tumors transsphenoidal approach with neuroendoscopy [11-14]. Based on anatomical study, we found that it is easy to get to the sellar region and observe the important structures clearly. There are several advantages of endoscopic endonasal transphenoidal surgery for anterior communicating artery aneurysm. Firstly, avoid scars caused by craniotomy. 
Secondly, no need to stretch or resect the brain. Thirdly, by neuroendoscopy we can clearly observe the optic nerve, optic chiasm, cisterna lamina terminalis, bilateral arteriae cerebri anterior, part of frontal lobe gyri rectus and the anterior communicating artery complex. So after blocking A1 of anterior cerebral artery, we can clearly see the anterior communicating artery complex and clip the aneurysm safely. But there are also some problems of the endoscopic endonasal transphenoidal surgery for clipping of anterior communicating artery aneurysm. Firstly, Postoperative cerebrospinal fluid rhinorrhea: The closure of dura is very important in operation, despite the skull base reconstruction can prevent the occurrence of cerebrospinal fluid rhinorrhea [15], inevitably some patients still have this complication. For these patients, we can take some measures, such as lumbosacral cerebrospinal fluid external drainage, strengthen antiinfective therapy, most of them can be cured. If these treatments were ineffective, cerebrospinal fluid rhinorrhea repair can be considered. Secondly, select indications for surgery strictly. If the top of anterior communicating artery aneurysm points to anterior inferior, this surgical approach can get to the top of anterior communicating artery aneurysm firstly, so it is difficult to control bleeding when the aneurysm rupture. If the top of anterior communicating artery aneurysm points to posterosuperior, it is difficult to see the surrounding vital structures of aneurysm. If decompressive craniectomy or haematoma elimination is needed, we should consider carefully in accordance with the patient. Thirdly, it is difficult to control bleeding, so cooperation and special equipments should be required in surgery. Such as special plier can help place aneurysm clip on it, and endoscope holder can help liberate the hands of operator.

\section{Conclusion}

In the anatomical research we find that carotid artery and its branch ophthalmic artery can be seen clearly if the base of the skull bone window can be expanded appropriately. Some literatures have reported the feasibility of ophthalmic artery aneurysm clipping with neuroendoscopy by endonasal transsphenoidal approach [16]. Selecting the indication for surgery is very important: the directing of aneurysm, also if decompressive craniectomy or haematoma elimination is needed, we should consider carefully in accordance with the patient. So this operation approach needs further study and exploration to clinical application, in order to become a mature approach of anterior communicating artery aneurysm surgery. The study provide anatomical basis for anterior communicating aneurysm surgery by endonasal transphenoidal approach with neuroendoscopy so that it can be applied to clinical widely.
Acknowledgements

Not applicable.

Funding

I have no funding for the research.

Availability of data and material

Not applicable.

\section{Authors' contributions}

JM carried out the anatomical study and draft the manuscript. ZW conceived of the study, and participated in its design and coordination and helped to revise the manuscript. NZ and SL participated in the anatomical study. DJ and HC participated in statistical analysis. All authors read and approved the final manuscript.

\section{Competing interests}

The authors declare that they have no competing interests.

Consent for publication

Not applicable.

Ethics approval and consent to participate

Ethical approval for this study was obtained from Soochow Kowloon Hospital ethics committee.

\section{Author details}

${ }^{1}$ Department of Neurosurgery, Suzhou Kowloon Hospital Affiliated Shanghai Jiao Tong University, Suzhou 266021, China. ${ }^{2}$ Department of Otolaryngology, Affiliated Hospital of Qingdao University Medical Colledge, Qingdao 266003, China. ${ }^{3}$ Department of Neurosurgery, Chinese Medicine Hospital of Gaomi, Gaomi 261500, China.

Received: 2 July 2015 Accepted: 29 June 2016

Published online: 07 September 2016

\section{References}

1. Drazin D, Zhuang L, Schievink WI, Mamelak AN. Expanded endonasal approach for the clipping of a ruptured basilar aneurysm and feeding artery to a cerebellar arteriovenous malformation [J]. Clin Neurosci. 2012;19(1):144-8.

2. Fischer $\mathrm{G}$, Oertel J, Perneczky A. Endoscopy in aneurysm surgery [J]. Neurosurgery. 2012;70(2 Suppl Operative):184-90.

3. Gruber A, Dorfer C, Standhardt H, Bavinzski G, Knosp E. Prospective comparison of intraoperative vascular monitoring technologies during cerebral aneurysm surgery. Neurosurgery. 2011;68(3):657-73.

4. Andaluz N, Zuccarello M. Fenestration of the lamina terminalis as a valuable adjunct in aneurysm surgery [J]. Neurosurgery. 2004:55(5):1050-9.

5. Sekhar LN, Kalia KK, Yonas H, et al. Cranial base approaches to intracranial aneurysms in the subarachnoid space [J]. Neurosurgery. 1994;35(3):472-83.

6. Sengapta RP. Sugical management of anterior cerebral and anterior communicating artery aneurysms. In: Schmidekeds $\mathrm{H}$, editor. Operative Neurosurgical Techniques. Pennsylvania: Saunders; 2002. p. I181-1204.

7. Wang Z, Zhou D, et al. Surgical treatment of intracranial aneurysms and prevention of cerebral vasospasm [J]. Neurosurgical Disease. Research. 2005;4(1):13-5.

8. Song $M$, Zong $X$, Wang $X$, et al. Anatomic study of the anterior skull base via an endoscopic transnasal approach [J]. Clinical Neurology and Neurosurgery. 2011;113(4):281-4.

9. Li J, Zhao J, Wang S, et al. Surgical treatment and type of anterior communicating artery aneurysm [J]. Chinese Journal of Clinical Neurosurgery. 2008;13(9):513-6.

10. Ciric I, Ragin A, et al. Complications of transsphenoidal surgery:results of a national survey, review of the literature, and personal experience [J]. Neurosurgery. 1997;40:225-37.

11. Zhang $X, \mathrm{Hu} F, \mathrm{Gu} H$. Resection of craniopharyngioma in third ventricle endonasal transsphenoidal approach with neuroendoscopy [J]. Chinese Clinical Medicine. 2010;17(4):474-6.

12. Lu X, Wang Q, Ji W, et al. Resection of tuberculum sellae meningioma endonasal transsphenoidal approach with neuroendoscopy [J]. Chinese Journal of Neuromedicine. 2005;10(4):1045-8. 
13. Bhatki AM, Pant $\mathrm{H}$, Synderman $\mathrm{CH}$, et al. The expanded endonasal approach for the treatment of anterior skull base tumors. Oper Tech Otolaryngol. 2010;21:66-73

14. Dave SP, Bared A, Casiano PR. Surgical outcomes and safety of transnasal endoscopic resection for anterior skull tumors. Otolaryngol Head Neck Surg. 2007:136:920-7.

15. Zanation AM, Snyderman CH, Carrau RL, et al. Minimally invasive endoscopic pericranial flap:a new method for endonasal skull base reconstruction []]. Laryngoscope. 2009;119:13-8.

16. Wang Z, Ding X, Qin S, et al. Anatomical study on endoscopic endonasal transphenoidal surgery for ophthalmic artery aneurysm. Chinese Journal of Neurosurgery. 2003;19(6):418-20.

Submit your next manuscript to BioMed Central and we will help you at every step:

- We accept pre-submission inquiries

- Our selector tool helps you to find the most relevant journal

- We provide round the clock customer support

- Convenient online submission

- Thorough peer review

- Inclusion in PubMed and all major indexing services

- Maximum visibility for your research

Submit your manuscript at www.biomedcentral.com/submit
Biomed Central 